\title{
Community, Family, and Partner-Related Stigma Experienced by Pregnant and Postpartum Women with HIV in Ho Chi Minh City, Vietnam
}

\author{
Deborah Bain Brickley • Dang Le Dung Hanh • \\ Luu Thi Nguyet · Jeffrey S. Mandel • \\ Le Truong Giang • Annette H. Sohn
}

Published online: 16 December 2008

(C) The Author(s) 2008. This article is published with open access at Springerlink.com

\begin{abstract}
Pregnant and postpartum women with HIV often face stigma and discrimination at home and in the community. In Vietnam, associations between HIV and the "social evils" of drug use and sex work contribute to stigmatization of people with HIV. We conducted a qualitative study to explore discrimination experienced by HIV-positive pregnant and postpartum women in Ho Chi Minh City at home and in the community. We conducted 20 in-depth interviews and two focus group discussions. Participants described managing disclosure of their HIV infection because of fear of stigma and discrimination, particularly to the wider community. In cases where their HIV status was disclosed, women experienced both discrimination and support. The findings highlight the need for targeted interventions to support pregnant and postpartum women with HIV, particularly during this period when they are connected to the healthcare system and more readily available for counseling.
\end{abstract}

D. B. Brickley $(\bowtie) \cdot$ L. T. Nguyet · J. S. Mandel · A. H. Sohn Global Health Sciences, University of California, San Francisco, CA, USA

e-mail: dbain@psg.ucsf.edu

D. Le Dung Hanh

Hung Vuong Obstetrics Hospital, Ho Chi Minh City, Vietnam

L. T. Giang

Ho Chi Minh City Provincial AIDS Committee,

Ho Chi Minh City, Vietnam

A. H. Sohn

Division of Pediatric Infectious Diseases,

University of California, San Francisco, CA, USA
Keywords Stigma - Discrimination · HIV/AIDS .

Pregnancy $\cdot$ Postpartum $\cdot$ Vietnam

\section{Introduction}

Stigma related to human immunodeficiency virus (HIV) infection can be devastating in the lives of people infected with and affected by the disease. Women are particularly vulnerable to HIV stigma and its consequences because of existing gender-related imbalances in social, cultural, and economic power structures. Given that HIV is associated with sex and the expression of sexuality, and that male sexuality historically has been seen as more acceptable in most societies (Bunting 1996), women are more likely to be treated as morally responsible for their infection status. Additionally, the lack of communities of support for women with HIV, particularly in resource-limited settings, means that they may find themselves coping with HIVrelated stigma alone. This situation is especially challenging during pregnancy and postpartum, when women may be preoccupied not only with the physical and psychological effects of having HIV, but also with preventing HIV transmission to their infants and/or avoiding disclosure of their HIV status to their families and communities.

The government of Vietnam has identified prevention of mother-to-child transmission (PMTCT) of HIV as a national priority (Vietnam Prime Minister, 2004), and PMTCT programs began operating in 2004 in four provinces, including Ho Chi Minh City. Recent evidence indicates that the reported cases of HIV among women in Vietnam have been underestimated (Nguyen et al. 2008; Analysis and Advocacy Report 2007). Whereas in 1996 HIV prevalence was $0.04 \%$, national sentinel surveillance from 2005 showed that $0.37 \%$ of antenatal women in 
Vietnam were infected with HIV (Nguyen et al. 2008), with a prevalence of $0.6 \%$ in Ho Chi Minh City (Vietnam Ministry of Health, National Institute of Hygiene and Epidemiology 2005). An estimated 7,000-8,000 women with HIV gave birth in 2000 (Socialist Republic of Vietnam 2005).

Addressing HIV stigma has become an urgent issue in Vietnam as the government and its international partners scale up prevention and treatment programs for people living with HIV and acquired immunodeficiency syndrome (AIDS). There is concern among public health advocates that the social stigma of AIDS will negatively affect access to these programs. Exacerbating the challenge has been the linkage of HIV to drug use and sex work, which are officially known as "social evils." Most public HIV education has used "fear-based messaging," with dramatic representations of bloody syringes, prostitutes, skulls, and coffins to portray the dangers (i.e., HIV infection) of engaging in social evils (Hong et al. 2004). This linkage was institutionalized through government agencies that had a mandate to address both issues, such as the Central Department of Social Evils Prevention and the Provincial Steering Committees for AIDS, Sex Work and Drug Control Influence.

Women in Asia, in particular, often define themselves by how they fulfill their social and familial roles (Desai 1996; Dwyer et al. 1988). Vietnamese society is heavily influenced by the Confucian tradition of having the senior members of the family centrally involved in making decisions. As such, the entire family shapes a couple's decision to have children or to end a pregnancy, with particular pressure to have male offspring (Oosterhoff et al. 2008). Childbearing women in Vietnam may be disempowered by their immediate and in-law families with regards to childrearing decisions and the ability and frequency with which they seek health care. This situation is complicated further by the HIV-positive status of the woman and her partner, which may lead to blame, fear, and further disempowerment.

To improve uptake and efficacy, PMTCT programs should consider the social issues that these women face in their daily lives. Unfortunately, research exploring the reproductive experiences of women with HIV in Vietnam has been limited. We conducted a study to explore the experiences of pregnant and postpartum women with HIV infection at home and in the community. A separate objective was to explore these women's experiences in the health care setting (data not yet reported). We sought to examine women's experiences of stigma, factors influencing disclosure of HIV status, and their existing support networks and to identify opportunities for reducing stigma experienced by women with HIV in Vietnam.

\section{Methods}

Setting

The study was conducted between September 2005 and January 2006 in Ho Chi Minh City, Vietnam. The primary study site was Hung Vuong Obstetrics Hospital, a 900-bed tertiary-care hospital that serves as a major referral center for southern Vietnam.

\section{Study Participants and Recruitment}

Study participants were drawn from pregnant and postpartum women with HIV at antenatal care and infant PMTCT follow-up clinics and from HIV support groups in the city. They were invited to participate by study investigators and were provided with an informational letter. Participants gave verbal consent following review of the study objectives and procedures with a study staff member. Participants were sampled initially using stratified purposeful sampling to identify information-rich cases (Coyne 1997; Patton 2002) and then with emergent sampling, which allows investigators to follow promising leads based on emerging findings of the study (Patton 2002).

At the time of the study, the local PMTCT program included initiation of antiretroviral prophylaxis at 3436 weeks of pregnancy. Women in the study who had delivered prior to the expansion of the PMTCT program, however, did not access antiretroviral prophylaxis or had received only a single dose of nevirapine at delivery. Women who delivered at private and community hospitals also were unlikely to have had access to antiretroviral prophylaxis due to limited PMTCT program coverage. Some of the younger children of the postpartum women were participating in an infant follow-up study program, where they received early infant diagnostic testing.

\section{Study Design}

Data were collected through in-depth, semi-structured interviews and focus group discussions. Interviews were initially conducted to explore the main themes of the study. Focus group discussions then were held to validate and further explore data collected during the interviews (Morgan 1993) in "member checks," in which participants verified or denied the validity of preliminary data (Lincoln and Guba 1985).

\section{Study Implementation}

Interviewers and focus group facilitators were Vietnamese women counselors trained by the research team. 
A semi-structured interview guide was used to explore the following domains:

- HIV testing - why and when women decided to get tested, whether it was their choice, if they received counseling, and their overall experience with HIV testing

- accessing health care-factors influencing their choices of health care sites, both during their pregnancies and for their personal HIV care, and experiences in seeking health care

- abortion-whether they had considered or been counseled to abort, and family members' feelings about having the baby or not

- disclosure of HIV status-to whom they had and had not disclosed their HIV status, and factors influencing disclosure

- future interventions-programs or interventions that participants felt would improve the situation of pregnant and postpartum women with HIV.

A focus group discussion guide was developed by the study team after preliminary analysis of the individual interview data. All study participants were offered a travel reimbursement of 100,000 Vietnam Dong (approximately US\$7) for their participation.

\section{Data Management and Analysis}

Interviews and focus groups were digitally recorded, then transcribed in Vietnamese and translated into English. For quality control, a bilingual member of the research team reviewed a random section of all transcripts and compared them to the original audio recordings to ensure accuracy. The bulk of the analysis was conducted jointly by two study team members, one Vietnamese-speaking and one English-speaking. Inconsistencies in interpreting linguistic and cultural differences were clarified among study team members throughout the data analysis process.

Thematic analysis, a process of categorizing text and identifying associations between categories, was used to analyze the transcripts (Boyatzis 1998). Data analysts used ATLAS.ti version 5 qualitative analysis software (ATLAS.ti Scientific Software Development GmbH, Berlin) to code the transcripts and identify common themes. The results presented in this paper focus on the experiences of women with HIV within their families and communities.

\section{Protection of Human Subjects}

The study protocol was approved by the Committee on Human Research at the University of California, San Francisco and the Institutional Review Board of the Ho Chi Minh City Provincial AIDS Committee (FWA00003315).
All participants provided verbal informed consent in Vietnamese. Personal identifying information was not collected from study participants, and if names or other identifying information were mentioned during interviews and focus group discussions, they were deleted from the transcripts.

\section{Results}

We conducted a total of 20 interviews with women with HIV. Six women $(30 \%)$ were pregnant at the time of the interview, $13(65 \%)$ were postpartum, and $1(5 \%)$ had had an abortion (see Table 1). The age range of participants was 19-34 years. Four of the women (20\%) reported being HIV serodiscordant with their current sexual partner; 12 $(60 \%)$ were seroconcordant; and $4(20 \%)$ did not know or did not report their partner's serostatus.

We also conducted two focus group discussions with seven participants each. Three of the focus group participants had also participated in in-depth interviews.

Table 1 Characteristics of pregnant and postpartum participants with HIV

\begin{tabular}{lcl}
\hline Characteristics & $\begin{array}{l}\text { Interview } \\
\text { participants } \\
\text { Number }(\%)\end{array}$ & $\begin{array}{l}\text { Focus group } \\
\text { participants } \\
\text { Number }(\%)\end{array}$ \\
\hline Total & $20(100)$ & $14(100)$ \\
Age (years) & $9(45)$ & $9(64)$ \\
$19-24$ & $8(40)$ & $5(36)$ \\
$25-29$ & $3(15)$ & 0 \\
$30-34$ & & \\
Pregnancy status & $6(30)$ & 0 \\
Pregnant & $13(65)$ & $14(100)$ \\
Postpartum & $1(5)$ & 0 \\
Aborted & $6(46)$ & $9(64)$ \\
Age of infant at the time of study participation $(n=13)$ \\
1-3 months & $5(39)$ & $3(21)$ \\
$4-6$ months & $2(15)$ & $2(14)$ \\
$7-10$ months & & \\
Timing of HIV diagnosis & $7(35)$ & NA \\
Before pregnancy & $9(45)$ & NA \\
During pregnancy & $4(20)$ & NA \\
At or around delivery & $4(20)$ & NA \\
Partners' HIV status & $12(60)$ & NA \\
Serodiscordant & $4(20)$ & NA \\
Seroconcordant & & \\
Did not report or did not know & & \\
\hline
\end{tabular}

$N A$ not available because these questions were not asked of focus group participants 
Almost all study participants described experiencing stigma and discrimination at home, in the community, or both. We organized the results from in-depth interviews and focus groups around the three primary relationships through which women experienced stigma and discrimination: community, family, and partner.

\section{Community}

Study participants primarily described fearing stigma and discrimination from the community based on moral judgments about how they may have become infected with HIV. This led them to avoid disclosing their HIV status outside their immediate families. One study participant describes the effect that government propaganda about "social evils" has had on the general population:

"There was a program recently propagandizing social evils. They had an AIDS ghost character and pumped the air in it to make it bigger, with thorns all over. I didn't know [my HIV test] result at that time, and I felt cold. Wearing all death's heads and skulls! Now, I'm ill and I'm feeling hurt. I agree with propaganda for society, to help people who don't inject drugs to avoid HIV. But for those who are ill, they will feel an inferiority complex." [28-year-old pregnant woman, W07]

These powerful images are meant to instill a fear of engaging in high-risk behaviors, but they also link people living with HIV to "social evils." This has led to negative moral judgments against people with HIV, as this woman describes:

"I believe that they think only bad people are associated with this kind of disease. They must have done something shameful to be infected. Most of these people [with HIV] are injecting drug users or sex workers. It's quite predictable that infected people will be looked down upon." [23-year-old postpartum woman, W14]

Study participants described few examples of discrimination from community members, in large part due to lack of disclosure outside the family. One woman described her fear of what others would think of her if they found out she was infected with HIV:

"Who knows what people have in their minds. They might think that I did something immoral and that I have to suffer for my sin. With that thought in mind, my husband and I decided to keep silent." [26-yearold pregnant woman, W10]

Another woman also was afraid of being criticized socially:
"Patients [with HIV] are perpetually afraid their family, neighbors, and friends will know they have contracted this horrible disease. This disease is strongly associated with social evils and debauchery and is subject to social criticism. That's why people are scared of it." [23-year-old postpartum woman, W14]

Fear of discrimination often was founded in having witnessed discrimination against other people with HIV. The fear of community members finding out about her HIV status was so strong for one woman that she admitted to contemplating suicide:

"I have told myself I will never let anyone know about my disease. If there is effective medicine for the disease in 1 or 2 years, it's good for me. But if the situation gets worse, I'll commit suicide. I can't let people know about it. I feel too ashamed, too inferior. I see the infected people who are released from prison facing discrimination by my neighbors, and I felt bad about myself and left. I am sad when I think about it. I pray for a cure to come soon." [20-year-old postpartum woman, W20]

One of the few examples of actual discrimination from the community came from a woman who was active in the support community for people with HIV and whose HIV status was known by many. She described having to move repeatedly because her landlords would not accept her as a tenant due to her HIV status:

"Then [my neighbor] told the landlady, 'She has the same disease as mine. What a pity for her. Let her stay!' The landlady said, 'My God! You have the illness and you want to bring SIDA to my house! No, no. This month only. Next month, move out to another place.' I stayed there for half a month, then I moved out." [23-year-old postpartum woman, W11]

This participant was unique because she had been infected for many years and her HIV status was widely known. She had experienced a great deal of discrimination from both her family and her community, and yet she simultaneously had a support system, including her mother, her HIV-positive husband, and a support group for people with HIV that she had been involved with for many years.

Family

Women with HIV in our study reported choosing whether or not to disclose their HIV status to family members based on how they thought the information would be received. Because most of the participants were in stable health, those who chose to were usually able to hide their infection 
status and thus control any inadvertent disclosure. Several women, however, spoke of their fear of family members finding out about their HIV status from hospital staff even if they chose not to disclose their status themselves.

Study participants gave two primary reasons for not disclosing their HIV status to family members. First, participants expressed concern about causing emotional trauma to family members. One woman described how the news of her HIV infection might affect her mother:

"I'll keep my mouth shut as long as possible. I can't confront my mother with this deadly news. My mother is old, and I don't know if the news would destroy her completely." [26-year-old pregnant woman, W10]

Second, many participants worried about being the object of HIV stigma and discrimination, and in particular that their children could face discrimination whether or not they were also infected.

"Our fear is stigma and discrimination against our child. We will go to extremes to prevent that from happening. We will never let anyone in our family know anything about us." [26-year-old pregnant woman, W10]

Several women were concerned that family members would discover their HIV status after the woman's death. One woman, whose husband was HIV-uninfected, was concerned that if her baby was HIV-infected, others would find out about her HIV status:

"I am scared that my baby will be infected, that my two families will know. If the baby is uninfected, I have nothing to worry about. My husband said if the baby is uninfected, when I die, he will tell them I died because of some other kind of disease, not HIV." [25year-old pregnant woman, W08]

She, like many women in the study, preferred that her family and community think that she died of a disease other than AIDS. A reason for lying about the cause of death was to protect the husband-if he was still alive_-and the child from discrimination.

Many women described facing discrimination from family members who knew of their HIV status. This woman described the reactions of her father and sister:

“My father said that I'm a 'SIDA person,' therefore he didn't attend my wedding. Each time he came to my house, he expressed antipathy. In fact, [my HIVpositive husband and I] feel an inferiority complex, so I stopped going to his house. And my younger sister, she hates me and my younger [HIV-positive] brother very much, and she is afraid of AIDS when coming to $[\mathrm{my}]$ house." [23-year-old postpartum woman, W11]

Fear of HIV transmission through casual contact often manifested in isolating behaviors by family members toward the person with HIV, including separating household items (e.g., eating utensils, laundry) and in some cases physically isolating the woman with HIV. This is illustrated by one participant who described the way she and her HIV-infected husband were treated by his family, with whom they lived:

"Everything was separated. We have a double line clothes rack. My husband's and my clothes were on one line. His parents' and brother's clothes were on the other. Everything was kept apart. [My mother-inlaw] didn't let me touch the food or the dishes. Their things and ours were kept separated. I was so upset." [25-year-old woman who had an abortion, W12]

Many study participants were new mothers, and family members' fears of becoming infected or of the child becoming infected often had a deep effect on the women's relationships with their new babies. None of the postpartum participants were breastfeeding. Yet, in some cases, women were not allowed to directly care for their babies, as experienced by two women in the study who were separated from their babies by their mothers-in-law. One of these mothers reported:

"Since he was born, my baby has been looked after by my mother-in-law. She doesn't let me do it. She is scared. The baby can be infected from the mother, so she is afraid that if I take care of the baby, there will be more risk of him contracting the disease. She has been with the baby since he was born. I just help wash up or do the vegetables." [23-year-old postpartum woman, W19]

The woman went on to explain that her mother-in-law discriminated against her in other ways, such as in not allowing her to sit in the same chairs as others in the house.

In several cases, the participants and their partners did not live with the partner's parents. For one participant in this situation, her baby, once born, was taken away by the mother-in-law to live with her:

“After my labor, my mother-in-law said, 'If you keep him, you may infect him.' Then she kept the baby, and I wasn't allowed to do so. Since the baby was born, I haven't been allowed to hold him. She doesn't allow him to live with me. During my labor, she didn't visit. After I gave birth, she sent her niece to visit us and take [the baby] to my mother-in-law's family." [23-year-old postpartum woman, W11] 
She later described how she perceived her mother-inlaw's behavior as discriminatory:

“My mother-in-law discriminates. They don't let me touch my baby. I have to stay away and can only look at my baby. I can't touch or talk to him. They don't want to see me. They don't want me to visit my baby. They do everything to make me leave." [23-year-old postpartum woman, W11]

Interestingly, later in the interview the same participant seemed resigned that it was best for others to take care of her baby, since she felt she was not healthy enough to do so.

It should be noted that it was not only family members who feared HIV transmission from the mother to the baby; several women discussed their own concerns about infecting their babies through casual contact. Their intentions to protect their babies often resulted in the decision to be physically separated, as this woman describes:

"I didn't know whether I passed the illness to my baby or not. Since then, I always kept a distance from my baby. I didn't know whether I would pass the illness to my baby. What I knew was to keep a distance from the baby so that it wouldn't have the same illness. My baby should be first." [29-year-old postpartum woman, W15]

At the same time, several of the women described receiving support from their families, particularly from their own mothers, as illustrated by this woman:

"My mother loves me dearly. She will never turn her back on her own daughter. I decided to disclose [my HIV status] to my mother because she was my caregiver while I was in the hospital. I warned her so that she wouldn't get infected from me." [23-year-old postpartum woman, W14]

\section{Partners}

Participants in our study rarely reported discrimination from their male partners, even though in most cases, the partner knew the woman's HIV status. In fact, most participants reported that their male partners were emotionally supportive through the difficult experience of pregnancy and motherhood. This was generally the case even when the woman's partner was HIV-seronegative, as this woman describes of her husband:

"Sometimes I told myself not to make him unhappy. Sometimes, I told him 'I want to divorce. One person is enough and I should be the one who suffers. I don't want my children to suffer. As you tested [HIV] negative, we should divorce. If we don't, then maybe sometime in the future, you may accidentally be infected, and I will feel very guilty!' But he didn't agree. He said, 'If we die, then we die together. I don't want you to suffer by yourself." " [29-year-old postpartum woman, W15]

With support from partners, these women were able to focus on taking care of themselves and their babies. Concern about the baby's future was a common motivating factor for taking care of their own health. This woman described how she and her husband were receiving treatment for HIV in order to help their baby:

"We will try and do everything for [the baby's] future. We promised one another that we will try for our baby, not for ourselves, but we still have to get treatment. We're getting treatment to have better health in order to earn money and save up for our baby." [28-year-old pregnant woman, W07]

Yet in a few cases, participants described fear and discrimination from their partners. One woman reported that her husband (who was HIV-seronegative) discriminated against her because of his fear of HIV infection:

"[My husband] said that at [the] hospital, he was told transmission could occur via eating due to saliva-tosaliva contact. If there are scratches in your mouth, there will be blood in your saliva and therefore the disease will be transmitted. Therefore, for each meal, he had the food served for the two of us separately. I don't feel emotionally bonded to him any more, no matter how much I love him." [22-year-old postpartum woman, W04]

Another woman perceived that her partner's support was dependent on the baby's HIV serostatus. That is, if the baby eventually tested positive for HIV, the partner would withdraw his support and possibly abandon the mother and baby:

"I hope to have a healthy baby, uninfected. [My husband] will be even nicer to me. If the baby is infected, I think he may change. He may marry another woman for a better life. But if I have a healthy baby, I think he will be very nice to me." [25-year-old pregnant woman, W08]

\section{Discussion}

Women's relationships with their communities, family members and partners contained two cross-cutting themes. Similar to findings by Hong et al. (2004), we found that 
HIV discrimination in Vietnam is a result of two related but different factors-fear of HIV transmission/infection and making moral judgments against people with HIV due to the association of the disease with the "social evils" of drug use and sex work. The discrimination experienced by pregnant and postpartum women with HIV in our study was most hurtful when it came from close family members or when it resulted in concrete hardships, such as losing access to their children. Participants also described receiving support from family members and being encouraged to take care of themselves for the sake of their babies.

Fear of HIV transmission through casual contact persists among people living with HIV and the larger community, despite existing public education campaigns and counseling programs. It was interesting to note that some women believed that the discrimination they experienced was justified in some way. Even women who were not breastfeeding expressed fear of ongoing transmission risk to their infants, and one woman willingly chose to give up her child to her family. These findings point to the need to clarify public education messages emphasizing that HIV transmission is not associated with casual contact, and that pregnant women with HIV are unlikely to transmit HIV to their infants if they take advantage of PMTCT programs.

Almost universally, women avoided disclosing their HIV status to anyone outside the family due to fear of discrimination. A notable exception was the participant who had been infected with HIV for many years and who was very open about her HIV status in her community. The discrimination she experienced directly, both from members of her family and within the community (she experienced eviction due to her HIV status), provide a justification for the fears expressed by other women with HIV. These fears continue to be reinforced by recent incidents of public discrimination, such as children being discriminated against at school (Viet Nam News 2007). Fear of discrimination can result in economic consequences; women with HIV and their partners may avoid seeking employment for fear of discrimination. It can also result in health consequences for women and their partners and children if people with HIV avoid seeking health care until emergencies arise. Finally, women with HIV may internalize their fear, which can lead to or exacerbate anxiety disorders or depression (Vanable et al. 2006). Undoubtedly, stigmatizing attitudes within the community penetrate family relationships as well, resulting in discrimination within the family, fear of disclosure to family members, or even community discrimination against HIVnegative family members of people infected with HIV.

Several of our study participants reported that stigma and discrimination based on moral judgments about their character were more likely to be perpetrated by community members and some family members, but less likely by their primary partners. In fact, the majority of women in the study described receiving their sole social support from their husbands and partners, even when the partner was HIV-seronegative. When supported by partners and family members, women had greater motivation to care for themselves because of the responsibility they felt toward their children and families.

The women in our study were very selective about disclosure of their HIV status to their family members, as stigma and discrimination could affect their own sense of identity and role within the family. Several women who had disclosed their HIV status to their families reported being disempowered by their in-laws with regard to childrearing and, in extreme situations, being denied access to their own children because of the family's fear that the children would become infected with HIV. These reports are similar to findings of Oosterhoff et al. (2008), in which the whole family was involved in decisions around a couple having children. The addition of HIV infection into the social dynamic of the Vietnamese family widens the power differential to the disadvantage of women with HIV. As a result, these women may be stripped of their traditional filial and maternal roles after they disclose their HIV status.

Women in our study described having a will to live and take care of their health as best as possible because of the responsibility they felt toward their children. Bunting and Seaton (1999) found similar results in their study of mothers with HIV in the United States, a factor they labeled "staying healthy for kids." They found that women's concern about the safety and health of their children motivated them to take care of themselves and adhere to drug treatment. This motivation could be used to develop interventions to encourage Vietnamese women with HIV to seek treatment, adhere to antiretroviral therapy, and take care of themselves and their families.

Our study findings point to the need for other types of specific interventions to provide targeted support to pregnant and postpartum women with HIV. The pregnancy and postpartum period are important times to access women, when they are connected to the health care systems and are more readily available for counseling on PMTCT, family planning, self-care, and self-empowerment. Such interventions could include basic and practical information on HIV transmission risks in general and within the home, and coping strategies for dealing with public and private discrimination. Integrating stigma-reduction programs into family and community-centered models for health care delivery and social support also would be culturally appropriate. Furthermore, because of the strong influence of family and community members in childrearing decisions, interventions to educate the broader public should be 
considered. These interventions could include public education campaigns or new laws and policies that would empower women with HIV to maintain or exert authority over their young children and discourage family members from taking children away from their HIV-positive parents.

This study had a number of limitations. Study participants were not randomly selected, and because of the small sample size, we describe experiences in a specific population at a specific point in time. As an example, no study participants reported domestic violence; it is likely that a recruitment strategy targeting women with specific types of domestic or peripartum experiences would uncover such cases. In addition, we had difficulty recruiting women who had opted for or felt they had been coerced into abortion, due to their reluctance to share their experiences. These topics would be better explored through more targeted research endeavors.

Stigma that pregnant and postpartum Vietnamese women with HIV experienced through their family and community relationships was a painful, daily reminder of their infection status. Minor domestic decisions regarding, for example, how to wash dishes sometimes escalated to the removal of infants from their mother's care, and separated women from what would normally be their core base of social support. Interventions that provide women with the knowledge to dispel misunderstandings about HIV transmission and with tools for self-advocacy may help women to cope better with the challenges of living with HIV in Vietnamese society.

Acknowledgments We would like to thank the Center for AIDS Prevention Studies for their support (CAPS Innovative Grant, P30 MH062246 center grant). Dr. Brickley received support from the University Fellowship at the University of California, Berkeley, the Public Health Alumni Association scholarships (courtesy of Helen and Mayhew Derryberry and William Griffiths), the DrPH Block Grant, the Wellness Award, and the Russell M. Grossman Medical Research Award. Dr. Sohn was supported by a grant from the National Institute of Child Health and Human Development, US National Institutes of Health (K23 HD047166). We would also like to thank our study participants, who were willing to share their stories.

Open Access This article is distributed under the terms of the Creative Commons Attribution Noncommercial License which permits any noncommercial use, distribution, and reproduction in any medium, provided the original author(s) and source are credited.

\section{References}

Analysis and Advocacy (A2) Report. (2007). The HIV Epidemic in Ho Chi Minh City: Where is it going? Retrieved January 15,
2008, from: http://www.fhi.org/NR/rdonlyres/epgt514eanmi373b a2ijqewgxsaxcrude6uiunko666b24yndvuex7tjcyvhc5b7544bljt2f pns5f/HIVepidemicHCMcenhv.pdf.

Boyatzis, R. E. (1998). Transforming qualitative information: Thematic analysis and code development. Thousand Oaks: Sage Publications.

Bunting, S. M. (1996). Sources of stigma associated with women with HIV. ANS. Advances in Nursing Science, 19(2), 64-73.

Bunting, S. M., \& Seaton, R. (1999). Health care participation of perinatal women with HIV: What helps and what gets in the way? Health Care for Women International, 20(6), 563-578. doi:10.1080/073993399245467.

Coyne, I. T. (1997). Sampling in qualitative research. Purposeful and theoretical sampling; merging or clear boundaries? Journal of Advanced Nursing, 26(3), 623-630. doi:10.1046/j.13652648.1997.t01-25-00999.x.

Desai, N. (1996). Women in Indian society. In A. M. Shah, B. S. Baviskar, \& E. A. Ramaswamy (Eds.), Social structure and change (Vol. 2). New Delhi: Sage Publications.

Dwyer, D. H., Bruce, J., \& Cain, M. (1988). Home divided: Women and income in the third world. Palo Alto: Stanford University Press.

Hong, K. T., Anh, N. T. V., \& Ogden, J. (2004). Understanding HIV and AIDS-related stigma and discrimination in Vietnam. Washington, DC: International Center for Research on Women.

Lincoln, Y. S., \& Guba, E. G. (1985). Naturalistic inquiry. Beverly Hills: Sage Publications, Inc.

Morgan, D. L. (1993). Successful focus groups: Advancing the state of the art. Newbury Park: Sage Publications, Inc.

Nguyen, T. A., Oosterhoff, P., Hardon, A., Tran, H. N., Coutinho, R. A., \& Wright, P. (2008). A hidden HIV epidemic among women in Vietnam. BMC Public Health, 8, 37. doi:10.1186/1471-24588-37.

Oosterhoff, P., Anh, N. T., Hanh, N. T., Yen, P. N., Wright, P., \& Hardon, A. (2008). Holding the line: Family responses to pregnancy and the desire for a child in the context of HIV in Vietnam. Culture, Health \& Sexuality, 10(4), 403-416. doi:10.1080/13691050801915192.

Patton, M. Q. (2002). Qualitative research and evaluation methods (3rd ed.). Thousand Oaks: Sage Publications, Inc.

Socialist Republic of Vietnam. (2005). Second country report on following up the implementation to the declaration of commitment on HIV/AIDS, January 2003-December 2005. Retrieved November 29, 2007, from http://www.unaids.org.vn/resource/ topic/natstrat/ungass_17jan06_e.pdf.

Vanable, P. A., Carey, M. P., Blair, D. C., \& Littlewood, R. A. (2006). Impact of HIV-related stigma on health behaviors and psychological adjustment among HIV-positive men and women. AIDS and Behavior, 10(5), 473-482. doi:10.1007/s10461-0069099-1.

Vietnam Ministry of Health, National Institute of Hygiene and Epidemiology. (2005). Household survey on HIV prevalence and AIDS indicators in Ho Chi Minh City and Thai Binh province. Hanoi: World Bank Project on HIV/AIDS Prevention and Control in Vietnam.

Viet Nam News. (2007, July 3). HIV prejudice ruins education (p. 5). Vietnam Prime Minister. (2004). Decision of the prime minister on approval of the national strategy on HIV/AIDS prevention and control in Viet Nam up to 2010 with a vision to 2020 (March 17 ed., Vol. 36/2004/QD-TTg, pp. 1-7). Hanoi. 